\title{
Editorial
}

\section{Enthusiasm, reality, and cost-effectiveness analysis}

The enthusiasm of researchers and clinical investigators is an admirable and perhaps necessary trait for performing good clinical research but must not obscure the interpretation of trial results. Cost-effectiveness analysis is being promoted as an objective means to measure the balance between the effectiveness of a treatment and its cost. Although cost-effectiveness analysis in medicine is in its infancy, much work has been performed to solidify its methodological underpinnings. ${ }^{1-3}$ Clinicians, nevertheless often remain suspicious of the process as they realise how difficult it may be to have reliable cost and efficacy information.

This issue contains an article by Aristides and colleagues on the cost-effectiveness of abciximab in preventing restenosis following percutaneous transluminal coronary angioplasty (PTCA). ${ }^{4}$ We agree wholeheartedly with the caveats expressed by Glasziou in his accompanying editorial $^{5}$ and add some further worries in this commentary.

The key ratio in cost-effectiveness analysis has a numerator of costs and a denominator that measures clinical efficacy such as years of life saved. In assessing the efficacy of an intervention it is necessary to consider critically the totality of the evidence. Aristides et al have relied only on the data from the 2009 patients enrolled in EPIC, ${ }^{67}$ although referring to the results of the more recent EPILOG $^{8}$ and CAPTURE ${ }^{9}$ trials to support their argument for the safety of abciximab. However, these two trials address a reasonably similar patient population to EPIC and therefore the 4057 patients included in these later trials could and should have been incorporated into the measure of efficacy.

In EPIC there was no mortality advantage nor any additional protection from myocardial infarction beyond what was observed at 30 days. The $8.1 \%$ absolute decrease in the composite end point at six months was largely due to a decrease in repeat PTCA $(20.9 \% v 14.4 \%)$. However, only US centres participated and the American proclivity for aggressive interventional cardiology is well appreciated..$^{10-12}$ For example, a recent population study of elderly postmyocardial infarction patients showed that PTCA is performed eight times more frequently in American than Canadian patients with no difference in mortality at one year. ${ }^{12}$ It is therefore quite possible that a soft end point, such as repeat PTCA, may be practice driven. The possibility of important geographic practice variations affecting outcomes, coupled with international variations of costs, invalidates the conclusion of Aristides et al that their economic results are transferable to other health care systems.

How can one reconcile the diverse clinical results? EPIC recruited patients from 1991 and recent improvements in patient selection and equipment, especially the introduction of stents, may have influenced the impact of abciximab. In addition, the maximum efficacy of abciximab in EPIC was observed in patients undergoing direct coronary atherectomy, ${ }^{13}$ a technique not widely used outside a few specialist centres.

The totality of this evidence thus suggests that abciximab following PTCA has no significant effect on mortality nor on repeat revascularisations at six months, but may decrease early myocardial infarction, particularly non-Q wave infarction, by about $40 \%$. These conclusions are supported by the prevailing evidence of the importance of platelets in thrombosis whereby early post-PTCA complications are due to platelet activation and subsequent aggregation. Restenosis is a more intermediate term complication resulting from vessel recoil and smooth muscle proliferation, while late complications are often due to disease progression in other vessels.

Aristides et al present economic analyses that are less than perfect; the estimate of clinical efficacy suffers from selection bias and the Markov model is also limited by the selective use of historical controls from the 1980s that is particularly inapplicable given the rapid advances in PTCA technology. To gain the trust of both physicians and administrators, the parameters used in this model need to be stated more explicitly. The decision tree for the model should be presented as well as the probabilities assigned to each branch.

The economic analysis relying exclusively on the EPIC data is more transparent but appears to have an accounting error. The incremental cost of $\$ 1054$ is reported to be associated with an incremental efficacy at six months of $8.1 \%$ to give a cost of $\$ 13012(1054 / 0.081)$ per patient free from a serious event. However, during the initial hospitalisation there were $4.5 \%$ fewer events with abciximab and the costs of these events had already been considered in calculating the incremental cost. Consequently, the incremental cost of $\$ 1054$ is actually associated with an incremental efficacy of $3.6 \%(8.1 \%-4.5 \%)$ giving a cost of $\$ 29000(1054 / 0.036)$ per patient free from a serious event.

Owing to these shortcomings, we present our own "back of the envelope" economic analysis. Current myocardial infarction rates following PTCA are approximately 3-5\% and if the efficacy of abciximab to reduce myocardial infarction is $40 \%$ then about two infarctions will be avoided for each 100 patients treated, at a total cost in the vicinity of $\$ 150000$. As a first approximation, the impact of myocardial infarction may reduce a patient's quality of life to 0.9 ( 1 being full health). If one assumes a 10 year survival following PTCA, one could expect that the prophylactic treatment of all high risk patients would add two quality adjusted life years (QALYs) at a cost of $\$ 75$ 000/QALY. By conventional technology assessment standards this would place abciximab in a relatively unfavourable position. ${ }^{14}$ However, the adoption of a more selective programme treating only patients with intraluminal thrombus identified at arteriography may be more cost effective. This approach is analogous to the increased cost effectiveness of secondary compared to primary prevention for hypercholesterolaemia.

Nevertheless the glycoprotein (GP) IIb/IIIa agents are an exciting class of drugs and the accumulation of further data may establish their importance in acute coronary syndromes. This role will likely vary in different patients in different health care systems. As more compounds are introduced it may be anticipated that costs will decrease. However, the true costs of the benefits of these drugs must be fully appreciated so that informed societal decisions may be taken. 
This commentary is meant to guard against "GP IIb/IIIa mania". Regarding economic analyses, we propose the systematic inclusion of all relevant studies to estimate best the parameters of interest. The assumptions and data employed in any mathematical model must be transparent and understandable to the clinicians and administrators who will use the analyses to guide their decisions. Extreme care must be exercised if one attempts to transfer costeffectiveness analysis across international borders. As with the general medical literature, readers must critically examine cost-effectiveness analysis for their applicability in their own environments.

Centre Hospitalier de l'Université de Montréal,

J M BROPHY

Pavillion Notre-Dame,

Montréal, Québec H2L 4M1, Canada

John Radcliffe Hospital,

P SLEIGHT

Oxford OX3 9DU, UK

1 Russell LB, Gold MR, Siegel JE, Daniels N, Weinstein MC. The role of cost-effectiveness analysis in health and medicine. Panel on costeffectiveness in health and medicine. $¥ A M A$ 1996;276:1172-7.

2 Weinstein MC, Siegel JE, Gold MR, Kamlet MS, Russell LB. Recommendations of the panel on cost-effectiveness in health and medicine. $7 A M A$ 1996;276:1253-8.

3 Siegel JE, Weinstein MC, Russell LB, Gold MR. Recommendations for reporting cost-effectiveness analyses. Panel on cost-effectiveness in health and medicine. $¥ A M A$ 1996;276:1339-41.
4 Aristides $M$, Gliksman M, Rajan N, Davey P. Effectiveness and cost effectiveness of single bolus treatment with abciximab (ReoPro) in preventing restenosis following percutaneous transluminal coronary angioplasty in high risk patients. Heart 1997;78:12-17.

5 Glasziou P. Using cost effectiveness for subsidy decisions. Heart 1997;78: $7-8$

6 The EPIC Investigators. Use of a monoclonal antibody directed against the platelet glycoprotein IIb/IIIa receptor in high risk angioplasty. $N$ Engl F Med 1994;330:956-61.

7 Topol EJ, Califf RM, Weisman HF, et al. Randomised trial of coronary intervention with antibody against platelet IIb/IIIa integrin for reduction of clinical restenosis: results at six months. The EPIC investigators. Lancet 1994;343:881-6.

8 The EPILOG Investigators. Platelet glycoprotein IIB/IIIa receptor blockade and low-dose heparin during percutaneous coronary revascularisation. $N$ Engl f Med 1997;336:1689-96.

9 The CAPTURE Investigators. Randomised placebo controlled trial of abciximab before and during coronary intervention in refractory unstable angina: the CAPTURE study. Lancet 1997;349:1429-35.

10 Rouleau JL, Moye LA, Pfeffer MA, et al. A comparison of management patterns after acute myocardial infarction in Canada and the United States. The SAVE investigators. N Engl f Med 1993;328:779-84.

11 Mark DB, Naylor CD, Hlatky MA, et al. Use of medical resources and quality of life after acute myocardial infarction in Canada and the United States. N Engl f Med 1994;331:1130-5.

12 Tu JV, Pashos CL, Naylor CD, et al. Use of cardiac procedures and outcomes in elderly patients with myocardial infarction in the United States and Canada. N Engl F Med 1997;336:1500-5.

13 Lefkovits J, Blankenship JC, Anderson KM, et al. Increased risk of non-Q wave myocardial infarction after directional atherectomy is platelet dependent: evidence from the epic trial. Evaluation of $\mathrm{c} 7 \mathrm{e} 3$ for the prevention of ischemic complications. F Am Coll Cardiol 1996;28:849-55.

14 Laupacis A, Feeny D, Detsky AS, Tugwell PX. How attractive does a new technology have to be to warrat adoption and utilization? Tentative guidelines for using clinical and economic evaluations. Can Med Assoc 7 1992;146:473-81.

\section{REBUTTAL}

\section{Murmurs from the heart (or why the stethoscope is not an economic tool)}

We thank Drs Sleight and Brophy for their challenging editorial on our ReoPro cost-effectiveness study. The editorial raises several important issues to which we would like to reply. In general, we agree that publication bias can exist with cost-effectiveness studies but remind our critics that this is a potential problem with all scientific research. Further, we accept that the long term outcomes of patients given ReoPro were unclear as we had only six month efficacy data. That was why we performed a two-step analysis with cost-effectiveness data from the pivotal study presented first, followed by an analysis to explore the impact on long term outcomes.

The trial based analysis is not flawed in its scope or because of selection bias. The EPIC trial was concerned with specific indications that were not fully examined in the other two studies mentioned (CAPTURE and EPILOG). Further, no accounting error has been made. The modelled analysis aimed to explore the long term outcomes with ReoPro and indicated cost-effective event free survival and overall survival. Three year follow up data of EPIC patients have since been published and have shown significant improvement in event free survival and, despite limited statistical power for individual end points, a trend to reduced mortality (odds ratio 0.78; 95\% confidence intervals (CI) 0.53 to 1.14 ).

We defend the focus on the EPIC trial and clarify that the results of the other clinical trials were considered at the time of writing (see "clinical effectiveness"). However, the aim was to examine cost-effectiveness in the EPIC defined high risk population. ${ }^{1}$ The EPILOG study essentially assessed an "all comers population" that excluded patients who were eligible for the EPIC study ${ }^{2}$ except patients with "EPIC eligible" morphological characteristics. The CAPTURE study assessed the use of ReoPro to stabilise refractory unstable angina patients before PTCA, administering a 24 hour treatment and stopping the ReoPro infusion soon after the PTCA. ${ }^{3}$ Consequently, these two studies were not similar to EPIC. Despite this, we mentioned in the article that these studies confirm the level of treatment effect. In addition, only the EPIC trial collected detailed resource use information available for analysis that made a trial based analysis possible. Safety was improved in EPILOG with more refined techniques in administration than seen in EPIC. Therefore, the main issue from the other studies was one of an improved risk:benefit ratio, which was discussed.

The value of reduced PTCA as a part of the composite clinical end point is questioned as the baseline level of PTCA may be too high. PTCA was recorded as an urgent intervention for a recurrent ischaemic event and was strictly defined. We contend that urgent PTCA is a relevant efficacy end point and appropriate for a trial based evaluation. Funders and clinicians can make up their own minds as to the value for money gained from the trial based evaluation. Further, PTCA was credibly measured within a large double blind randomised trial. Indeed, one of the best epidemiological studies in this area by Weintraub et al in the USA ${ }^{4}$ confirms that restenosis leads to greater myocardial infarction, repeat PTCA, and CABG. Although mortality was not reduced, this is linked to a high rate of 
repeat PTCA within the first year of restenosis (75\%). Consequently, such high rates of PTCA may prevent death after restenosis. We included epidemiological studies with intervention rates closer to that for Australia but raised the findings of Weintraub et al.

The applicability of the EPIC trial is necessarily limited to the procedures used and the patients studied, which can be said of any major clinical trial. Indeed, the use of stents may have changed the role of ReoPro but this question is being addressed in the EPILOG stent substudy. Data on the use of stents in high risk patients are limited and the onus is perhaps on our critics to present relevant data. We caution against subgroup analysis given its reduced statistical power and comparability between groups, and remind readers that the best estimate of treatment effect comes from the overall effect from a well conducted trial. This is why we were cautious of interim results indicating similar benefit in non-high risk elective PTCA (that is, a much wider group) and await further research.

Consequently, it is surprising that our critics do not accept the results of a large randomised trial on efficacy at six months in favour of an assessment of what might happen if the trial was run again. Interestingly, three year follow up results have since been published. ${ }^{5}$ While remembering that the EPIC trial was not powered to look for a mortality benefit, outcomes (as measured by the composite end point) were significantly improved up to three years after treatment and an increasing trend to reduced mortality exists (odds ratio $0.78 ; 95 \%$ CI 0.53 to 1.14 ).

The results of the model are presented separately to make the analysis transparent. As indicated in the Australian cost-effectiveness guidelines, a modelled economic evaluation should follow on from a trial based analysis so that the impact on results can be assessed. ${ }^{6}$ Necessarily, much of the technical detail could not be published in an article of this nature for which content and word limit is imposed. We admit that a limited number of cohort studies were included in the modelled analysis but this resulted mainly from including studies that assessed the long term impact of restenosis post-PTCA.

There is no accounting error in the calculation of the cost-effectiveness ratios. As is standard practice, the cumulative cost is related to the cumulative benefit over the six month period. For those who are interested, a standard text is recommended. ${ }^{7}$ On average, patients on ReoPro incurred an additional cost of $\$ 1054$ by six months and experienced an $8.1 \%$ reduction in the composite end point. Health events can occur in the denominator as "effective- ness" as well as in the numerator as "cost". There is no sense in removing the risk reduction during the initial hospitalisation from the six month cost-effectiveness ratio.

Finally, the "back of the envelope analysis" presented in the editorial seems to go against our critics' central message for good quality and comprehensive data analysis. We contend that our "envelope" was considerably larger but appreciate their "enthusiasm".

In conclusion, the trial based analysis presented considers a particular indication, does not suffer from selection bias, and has no accounting error. The applicability of the trial is indeed limited to its design (as with any trial) and issues such as the impact of stenting are not yet known (as was highlighted). We presented a modelled evaluation to assess the impact on long term outcomes. The need for a modelled approach requires a balance between its scope and foundations. The separate presentation of the trial based and model based analyses can assist the reader in appraising each. Since our modelling exercise, event free survival at three years has been confirmed in the EPIC follow up study with a trend to improved survival. This suggests to us that the "totality of evidence" is somewhat different.

M ARISTIDES M GLIKSMAN

$M-T A G$,

PO Box 5639,

NSW 2057, Australia

Eli Lilly Austalia

112 Wharf Road,

West Ryde,

NSW 2114, Australia

1 Topol EJ, Califf RM, Weisman HF, et al. Randomised trial of coronary intervention with antibody against iib/iiia integrin for reduction of clinical intervention with antibody against iib/iiia integrin for re
restenosis: results at six months. Lancet 1994;343:881-5.

2 The EPILOG Investigators. Platelet glycoprotein IIb/IIIa receptor blockade and low dose heparin during percutaneous coronary revascularisation. $N$ Engl f Med 1997;336:1889-96.

3 The CAPTURE Investigators. Randomised placebo controlled trial of abciximab before and during coronary intervention in refractory unstable angina: the CAPTURE study. Lancet 1997;349:1429-35.

4 Weintraub WS, Ghazzal ZMB, Douglas JS, et al. Long-term clinical follow-up in patients with angiographic restudy after successful angioplasty. Circulation 1993;87:572-82.

5 Topol EJ, Ferguson JJ, Weisman HF, et al. Long-term protection from myocardial ischemic events in a randomised trial of brief integrin $\beta_{3}$ blockade with percutaneous coronary intervention. $f A M A$ 1997;278:479-84.

6 Commonwealth of Australia. Guidelines for the pharmaceutical industry on preparation of submissions to the pharmaceutical benefits advisory committee: including submissions involving economic analyses. Canberra: Australian Government Printing Office, 1995.

7 Drummond MF, Stoddart GL, Torrance GW. Methods for the economic evaluation of health care programmes. Oxford: Oxford University Press, 1987: 48-53. 